\title{
Acknowledgement to Referees
}

(c) Springer Nature Switzerland AG 2021

\section{Dear Reader,}

Welcome to the final issue of BioDrugs for 2021.

I wish to reflect on this year's achievements, and to thank all those who have contributed their time and effort to guarantee the quality of the content published in the journal.

The high quality of content published in BioDrugs has been reflected in the most recent Impact Factor of 5.807 and CiteScore ${ }^{\mathrm{TM}}$ of 9.40. These place the journal in the first quartile for Pharmacology \& Pharmacy in Clarivate Analytics, and $13^{\text {th }}$ of 246 journals $\left(94^{\text {th }}\right.$ percentile) in Medicine - Pharmacology in Scopus. Further, BioDrugs has published content in a timely manner, with an average time from submission to acceptance of 86 days in 2021.

In 2021, over 48 articles have been published. The most popular of these in terms of downloads from SpringerLink have been:

Clinical Development of New Antibody-Drug Conjugates in Breast Cancer: To Infinity and Beyond

Immunogenicity Challenges Associated with Subcutaneous Delivery of Therapeutic Proteins

Comparability of Biologics: Global Principles, Evidentiary Consistency and Unrealized Reliance

Efficacy, Safety and Immunogenicity of MB02 (Bevacizumab Biosimilar) versus Reference Bevacizumab in Advanced Non-Small Cell Lung Cancer: A Randomized, Double-Blind, Phase III Study (STELLA)

Biologics and Targeted Synthetic Drugs Can Induce Immune-Mediated Glomerular Disorders in Patients with Rheumatic Diseases: An Updated Systematic Literature Review

Engineered Bacteriophage Therapeutics: Rationale, Challenges and Future

Efficacy, Safety, and Immunogenicity of HLX02 Compared with Reference Trastuzumab in Patients with Recurrent or Metastatic HER2-Positive Breast Cancer: A Randomized Phase III Equivalence Trial

Recent Achievements and Challenges in Prolonging the Serum Half-Lives of Therapeutic IgG Antibodies Through Fc Engineering

Safety of Intravitreal Gene Therapy for Treatment of Subjects with Leber Hereditary Optic Neuropathy due to Mutations in the Mitochondrial ND4 Gene: The REVEAL Study

Budget Impact Analysis of the Introduction of Rituximab and Trastuzumab Intravenous Biosimilars to EU-5 Markets

Social media sharing and other online mentions of information are other metrics with which to measure the reach of articles. This year the following articles published in BioDrugs scored highly on the Altmetric system:

Safety of Intravitreal Gene Therapy for Treatment of Subjects with Leber Hereditary Optic Neuropathy due to Mutations in the Mitochondrial ND4 Gene: The REVEAL Study

Clinical Development of New Antibody-Drug Conjugates in Breast Cancer: To Infinity and Beyond

Comparability of Biologics: Global Principles, Evidentiary Consistency and Unrealized Reliance

Administration of CD4+CD25highCD127-FoxP3+ Regulatory T Cells for Relapsing-Remitting Multiple Sclerosis: A Phase 1 Study

Efficacy, Safety, and Immunogenicity of HLX04 Versus Reference Bevacizumab in Combination with XELOX or mFOLFOX6 as First-Line Treatment for Metastatic Colorectal Cancer: Results of a Randomized, Double-Blind Phase III Study 
The COVID-19 pandemic has continued to present many challenges and I would like to thank all who have contributed to ensuring the journal has thrived despite the ongoing issues. Springer Nature has continued to support the global response to COVID-19 by making all relevant content immediately and freely available.

I would like to start by thanking the authors of the articles published in BioDrugs over the course of 2021. The enthusiasm of all authors for their chosen fields and their willingness to contribute content to the journal are crucial for its continued success.

The quality of published articles is also testament to the diligence of the peer reviewers. I would like to acknowledge the following individuals who acted as reviewers for BioDrugs in the last 12 months:

Mohammed I. Aladul, Iraq

Saja Alnahar, Jordan

Luca Antonioli, Italy

Kazuhiro Araki, Japan

Jon E. Arnason, USA

Raja Atreya, Germany

Yves Aubin, Canada

Albert J. Augustin, Germany

Sathy Balu-Iyer, USA

Liese Barbier, Belgium

Alain Beck, France

Shubha Bhat, USA

Francisco J. Blanco, Spain

Challice Bonifant, USA

Enrico Borrelli, Italy

John Botson, USA

Melissa Bowerman, UK

Jason Braithwaite, USA

Vitalis Briedis, Lithuania

Joseph Bubalo, USA

Myrna Candelaria, Mexico

Kuan Rong Chan, Singapore

Siew Eng Choon, Malaysia

Christos Chouaï, France

Beth Christian, USA

Laura C. Coates, UK

Arnon D. Cohen, Israel

Hillel P. Cohen, USA

Pier Conte, Italy

Esteban Cruz, Australia

Paola M. Cutroneo, Italy

Dimitrios Daoussis, Greece

Fernando de Mora, Spain

Joshua Drago, USA

Erwin Dreesen, Belgium

Niklas Ekman, Finland

Federica Facciotti, Italy

Napoleone Ferrara, USA

Josef Finsterer, Austria

Regina Folster-Holst, Germany

Massimo Franchini, Italy
Marco Franzetti, Italy

Egidio Freitas, Portugal

Inbar Friedrich Ben-Nun, USA

John P. Gabrielson, USA

Paul Gamlin, USA

Dayong Gao, USA

Roman Gardlik, Slovakia

Pere Gascon, Spain

Xin Ge, USA

Salah Ghabri, France

Clemens Giessen-Jung, Germany

Bente Glintborg, Denmark

Miguel Ángel Gonzalez-Gay, Spain

Boris Gorovits, USA

John Gubatan, USA

Luisa Guidi, Italy

Lora Hamuro, USA

Graham F. Hatfull, USA

Michael R. Hayden, Canada

Michael Hickey, Australia

Joachim Hombach, Switzerland

Xucheng Hou, USA

Li-feng Hsu, Taiwan, Republic of China

Huei-Kai Huang, Taiwan, Republic of China

Allison Hubel, USA

Jessica Jalbert, USA

Naoko Kanda, Japan

Tae Hyun Kang, Republic of Korea

Seoyoung C. Kim, USA

Masahiro Kino-oka, Japan

Allison Kolbe, USA

Hans-Christian Kolberg, Germany

Emanuela Lacana, USA

Edward Chia-Cheng Lai, Taiwan, Republic of China

Augustin Latourte, France

Mark G. Lebwohl, USA

Howard Lee, Republic of Korea

David S. Liao, USA

Michael N. Liebman, USA

Ruth Lopert, USA

Xiao-Yong Man, China 
Kenneth K.C. Man, UK

Marco Matucci-Cerinic, Italy

Matthew Mei, USA

Julie Meneghel, UK

Phillip Minar, USA

Lotte Minnema, The Netherlands

Thameez Mohammed Koyasseril Yehiya, USA

Thallachallour Mohanakumar, USA

Serafin Morales, Spain

Michael Morgan, Germany

Diane R. Mould, USA

Asuka Nanbo, Japan

Kyle O'Donnell, USA

Michael O'Dwyer, Ireland

Sonia T. Oskouei, USA

Konstantinos Papamichael, USA

Friedemann Paul, Germany

Jean-Phillipe Pellois, USA

Edith Pfister, USA

Stefano Piaserico, Italy

Diana P. Pires, Portugal

Elizabeth Price, UK

Varun K. Ranpariya, USA

Emanuel Raschi, Italy

Aymeric Ravel-Chapuis, Canada

Soledad Retamozo, Argentina

Kelly A. Reynolds, USA

Wolfgang F. Richter, Switzerland

Remy Robert, Australia

Giuseppe Roberto, Italy

Carlos A.V. Rodrigues, Portugal

Philip J. Rosenfeld, USA
Antonia P. Sagona, UK

Hironaga Satake, Japan

Martin Schiestl, Austria

Martin Schmelz, Germany

Giulia Scondotto, Italy

Ivana Stojanovic, Serbia

Kangho Suh, USA

Janet Sultana, Malta

Sasha Taleban, USA

Stephen J. Thomas, USA

Tiago Torres, Portugal

Tsen-Fang Tsai, Taiwan, Republic of China

Christopher J. Twelves, UK

Niels Vande Casteele, USA

Bram Verstockt, Belgium

Pablo Villoslada, Spain

Gianni Virgili, Italy

Philip Vlummens, Belgium

Arnold G. Vulto, The Netherlands

Norihiro Watanabe, USA

Keith Watson, UK

Christopher Webster, USA

Martina Weise, Germany

Valdemar Wendelboe Nielsen, Denmark

Astrid Wiens, Brazil

Edward Wild, UK

Elena Wolff-Holz, Germany

Charles C. Wykoff, USA

Toshinari Yamashita, Japan

Wei Zhang, China

Haiyan Zhou, UK

Zsombor Zrubka, Hungary

I am also very grateful to the members of the journal's Honorary Editorial Board, who have acted as peer reviewers and authors, and have provided guidance on journal content, policy and processes.

Lothar Bergmann, Klinikum der Johann Wolfgang Goethe-Universitat, Frankfurt, Germany

George D. Demetri, Dana Farber Cancer Institute, Boston, MA, USA

Terence R. Flotte, University of Massachusetts Medical School, Worcester, MA, USA

Timothy M. Ganey, Vivex Biomedical, Atlanta, GA, USA

Arnold Ganser, Hannover Medical School, Hannover, Germany

Hans-Peter Gerber, 3T Biosciences, Palo Alto, CA, USA

Thijs J. Giezen, Foundation Pharmacy for Hospitals in Haarlem, Haarlem, Netherlands

Bernd Groner, Georg-Speyer-Haus, Institute for Biomedical Research, Frankfurt, Germany

Francois Guilhot, Université de Poitiers, Poitiers, France

William H. Hildebrand, University of Oklahoma, Oklahoma City, OK, USA

Phillippe Moingeon, Servier Pharmaceuticals, Paris, France

Dafne Müller, Universitat Stuttgart, Stuttgart, Germany

Won Park, Inha University Medical School, Incheon, Republic of Korea

Cesar Picado, Hospital Clinic, Universitat de Barcelona, Barcelona, Spain

Thomas Schirrmann, YUMAB GmbH, Braunschweig, Germany

Susan Sharfstein, SUNY Polytechnic Institute, Albany, NY, USA 
Cy A. Stein, City of Hope Medical Center, Duarte, CA, USA

Raymond C. Stevens, University of Southern California, Los Angeles, CA, USA

William R. Strohl, BiStro Biotech Consulting, Bridgewater, NJ, USA

Bruce A. Sullenger, Duke University Medical Center, Durham, NC, USA

Jonathan Terrett, CRISPR Therapeutics, Inc., Cambridge, MA, USA

Gianluca Trifirò, University of Verona, Verona, Italy

Piotr Trzonkowski, Medical University of Gdansk, Gdansk, Poland

Jos W. van der Meer, Radboud University Medical Center, Nijmegen, The Netherlands

Gillian Woollett, Avalere Health, Washington, DC, USA

In terms of other important company initiatives, the Springer Nature 'Sustainable Development Goals (SDG) Programme' is aiming to connect researchers who are tackling the world's toughest challenges with practitioners in policy and business, while the 'Women in Science' initiative aims to help empower more women scientists to achieve scientific excellence. Finally, I am delighted to inform you that Springer Nature is now carbon neutral for its direct operations (offices, fleet and flights) and earlier this year signed The Climate Pledge, making a commitment to be Net Zero carbon by 2040.

Returning to BioDrugs, the editorial program for 2022 is well under way, and I am looking forward to bringing you many high-quality and authoritative articles in the field of biotechnology-based pharmaceuticals over the coming year.

I thank you for your continued support.

With best wishes,

Kathy Fraser, Editor 